\title{
LAWS IN NILPOTENT-BY-FINITE GROUPS
}

\author{
JOHN COSSEY ${ }^{1}$
}

1. A basic question in the theory of varieties of groups is whether the laws of every group are finitely based. Two classes of groups for which an affirmative answer has been given are the class of nilpotent groups (R. C. Lyndon [6]) and the class of finite groups (Sheila Oates and $M$. B. Powell [8]). In this paper we give an affirmative answer for a class of groups closely related to these two classes, namely, the class of groups for which some finite term of the upper central series has finite index. The proof of our main result uses the result of Oates and Powell, and also depends heavily on the method developed by L. G. Kovács and M. F. Newman in [5] to prove that result. Our notation follows that of [5].

Suppose that $w\left(x_{1}, \cdots, x_{n}\right)$ is any law. We define laws $w_{r}\left(x_{1}, \cdots, x_{n+r}\right)$ by

$$
\begin{aligned}
w_{0}\left(x_{1}, \cdots, x_{n}\right) & =w\left(x_{1}, \cdots, x_{n}\right) ; \\
w_{r}\left(x_{1}, \cdots, x_{n+r}\right) & =\left[w_{r-1}\left(x_{1}, \cdots, x_{n+r-1}\right), x_{n+r}\right], \quad r>0 .
\end{aligned}
$$

The variety defined by $w_{r}\left(x_{1}, \cdots, x_{n+r}\right)$ is denoted by $\mathfrak{B}_{r}$. We can now state our main result.

THEOREM 1. If $\mathfrak{W}_{0}$ can be generated by a single finite group, then for every integer $r \geqq 0$ there is an integer $s(>0)$ depending only on $w_{r}\left(x_{1}, \cdots, x_{n+r}\right)$ such that any subvariety of $\mathfrak{W}_{r}$ is generated by its s-generator free group.

This has the

COROLlary 1. Every subvariety of $\mathfrak{W}_{\text {r }}$ has a finite basis for its laws.

Proof. Suppose that some subvariety $\mathfrak{U}$ of $\mathfrak{W}_{r}$ does not have a finite basis for its laws. Then it is not difficult to see that the set of subvarieties of $\mathfrak{W}_{r}$ which have a finite basis for their laws does not satisfy the minimum condition (with respect to partial order by inclusion). From Theorem 1 we may conclude that the lattice of subvarieties of $\mathfrak{W}_{r}$ is the dual of the lattice of verbal subgroups of the free group $F_{s}$ of rank $s$ of $\mathfrak{W}_{r}$, and hence the verbal subgroups of $F_{s}$ do not satisfy the maximum condition. Since $\mathfrak{W}_{0}$ can be generated by a finite group, for every finitely generated free group $F$ of $\mathfrak{W}_{r}$,

Received by the editors March 1, 1967.

1 The author is a Fulbright-Hays scholar. 
$F / w_{0}(F)$ is finite [7, Theorem 14.2]. Also, it is clear that $w_{0}(F)$ is a finitely generated nilpotent group of class at most $r$. In particular then, $F_{s}$ is a finitely generated nilpotent-by-finite group, and so even satisfies the maximum condition for subgroups, a contradiction.

We can now also prove

Theorem 2. If $G$ is a group such that some finite term of the upper central series of $G$ has finite index, then there is a finite basis for the laws of $G$.

Proof. Suppose that the $r$ th term of the upper central series of $G$, $N$ say, has finite index. If we put $\operatorname{var}(G / N)$ for the variety generated by $G / N$, the theorem of Sheila Oates and M. B. Powell [8] tells us that $\operatorname{var}(G / N)$ has a finite basis for its laws. Let $w\left(x_{1}, \cdots, x_{n}\right)$ be a law defining $\operatorname{var}(G / N)$. Then clearly, $G$ satisfies the law $\left[w\left(x_{1}, \cdots, x_{n}\right), x_{n+1}, \cdots, x_{n+r}\right]$. The result now follows from Corollary 1.

As a special case of Theorem 2 we have

COROLLARY 2. If $\mathfrak{R}$ is a nilpotent variety and $\mathfrak{B}$ a variety generated by a finite group, then there is a finite basis for the laws of $\mathfrak{N} \cup \mathfrak{B}$.

Proof. Suppose $\mathfrak{V}$ is generated by the finite group $H$. Let $F$ be the free group of countably infinite rank of $\mathfrak{N}$. Then, putting $G=F \times H$, we have $\mathfrak{N} \cup \mathfrak{B}=\operatorname{var}(G)$, and $G$ clearly satisfies the hypotheses of the theorem.

COROLlary 3. Suppose that $G$ is a group with a normal subgroup $M$ of finite index such that some finite term of the lower central series of $M$ is finite. Then there is a finite basis for the laws of $G$.

Proof. Suppose that the $k$ th term of the lower central series of $M$ is finite. Then, by the corollary to Theorem 2 of [3], the $(2 k)$ th term of the upper central series of $G$ has finite index. The result then follows.

Finally, we observe without proof that the converse of Theorem 1 is true for nilpotent-by-finite groups. That is, if every subvariety of the variety generated by a nilpotent-by-finite group can be generated by a finitely generated group, then some finite term of the upper central series of that group has finite index.

2. There is only the proof of Theorem 1 remaining. We have observed in the proof of Corollary 1 that the finitely generated free groups of $\mathfrak{W}_{r}$ (and so of any subvariety of $\mathfrak{W}_{r}$ ) are nilpotent-by-finite groups and so are residually finite. From this it follows that any subvariety of $\mathfrak{W}_{r}$ is generated by its finite groups. But then it follows 
from a theorem of D. C. Cross [4, Theorem 1], that any subvariety of $\mathfrak{W}_{r}$ is generated by its critical groups. Thus, if we can put a bound on the numbers of generators of critical groups in $\mathfrak{W}_{r}$, we are finished.

Note that since $\mathfrak{S}_{0}$ can be generated by a finite group there exist integers $e, m, c$ such that [5, Corollary]

(i) the exponent of groups in $\mathfrak{B}_{0}$ divides $e$; (ii) every chief factor of groups in $\mathfrak{W}_{0}$ has order at most $m$; (iii) every nilpotent group in $\mathfrak{W}_{0}$ has class at most $c$.

We start with two trivial lemmas.

Lemma 1. If $G$ is a finite group in $\mathfrak{B}_{r}$ and $H / K$ is a chief factor of $G$ such that $H \leqq w(G)$, then $H / K \leqq Z(G / K)$, the centre of $G / K$.

Proof. Since $w_{0}(G)=w(G) \geqq H$ and $w_{r}(G)=1$, there is an integer $i$ such that $1 \leqq i \leqq r$ and $w_{i-1}(G) \cdot K \geqq H, w_{i}(G) \cap H \leqq K$. The result follows immediately.

Lemma 2. There is a bound on the class of nilpotent groups in $\mathfrak{B}_{r}$.

Proof. If $G$ is nilpotent, it follows immediately that the $(r+c+1)$ th term of the lower central series of $G$ is trivial.

Now let $G$ be a critical group in $\mathfrak{B}_{r}$. $w(G)$ is nilpotent, and so, if $w(G) \neq 1$, it must be a $p$-group for some prime $p$. There are three cases to consider:

(1) $w(G)=1$.

(2) $w(G) \neq 1, p$ divides $|w(G)|$, and $p$ divides $e$.

(3) $w(G) \neq 1, p$ divides $|w(G)|$, and $p$ does not divide $e$.

We deal with each case separately.

(1) Since $\mathfrak{W}_{0}$ can be generated by a finite group, there are only a finite number of critical groups in $\mathfrak{B}_{0}[8$, Theorem 3$]$ : hence if $w(G)=1, G \in \mathfrak{W}_{0}$ and there is a bound, $s_{1}$ say, on the number of generators of $G$.

(2) The proof of (2) is essentially a reproduction of the proof of $[5, \S 3]$; it is quite long and technical. We shall only indicate where the proof differs from that of [5] and refer the reader to [5] for details.

Let $G$ be critical, $F$ the Fitting subgroup and $\Phi$ the Frattini subgroup of $G$. If we can bound $|G / \Phi|$, we have a bound on the number of generators of $G$. Since $w(G)$ is nilpotent, $F \geqq w(G)$, and so $G / F \in \mathfrak{W}_{0}$. By $[1$, Theorems 1, 7] Gaschütz, $F / \Phi$ is a direct product of minimal normal subgroups $M_{1} / \Phi, \cdots, M_{l} / \Phi$ of $G / \Phi$, and $F / \Phi$ has a complement $L / \Phi$ in $G / \Phi$. It is an easy deduction from [8, Lemma 2.4.2] and Lemma 2 that $l \leqq c+r$. 
Observe that from (ii) and Lemma 1 either $\left|M_{i} / \Phi\right| \leqq m$ or $\left|M_{i} / \Phi\right|=p$. Since $p$ divides $e, p \leqq m$, and so $\left|M_{i} / \Phi\right| \leqq m, 1 \leqq i \leqq l$. Hence $|F / \Phi|$ is bounded.

To obtain a bound for the order of $L / \Phi$, the only modification to the argument of $[5]$ is that the series defined in the final paragraph of p. 532 is replaced by the following series:

$$
F=F_{0}>\cdots>F_{k}=w(G)>F_{k+1}>\cdots>F_{k+t}=1,
$$

where $F_{0}>\cdots>F_{k}$ is the series obtained by refining the lower central series of $F / w(G)$ with terms corresponding to the lower Frattini series of its factors; and $F_{k+i}=w_{i}(G), 0 \leqq i \leqq t$. Clearly $t \leqq r$. The proof of [5, Theorem 3.5] carries over for $0 \leqq i \leqq k$; and for $k<i \leqq k+t$, it is a consequence of the definition of $w_{r}$.

Suppose that $s_{2}$ is the bound on the number of generators of $G$ given by this method.

(3) Suppose that $G$ is critical. Since $p$ does not divide $e$, we have $w(G)=F$, the Fitting subgroup of $G$, and $F$ is complemented in $G$, by $H$ say. Now if $\Phi$ is the Frattini subgroup of $F$, then Lemma 1 gives us that $H$ acts trivially on $F / \Phi$. Thus $H$ centralizes $F[2$, Theorem 12.2.2], and so $H=1$. Hence $G$ is critical, nilpotent of class at most $r$ and so can be generated by $r$ elements [4, Theorem 8].

Now, put $s=\max \left(s_{1}, s_{2}, r\right)$, and we are finished.

\section{REFERENCES}

1. W. Gaschütz, Über die $\Phi$-Untergruppen endlicher Gruppen, Math. Z. 58 (1953), 160-170.

2. M. Hall, Jr., The theory of groups, Macmillan, New York, 1959.

3. P. Hall, Finite-by-nilpotent groups, Proc. Cambridge Philos. Soc. 52 (1956), 611-616.

4. Graham Higman, Identical relations in finite groups, pp. 93-100, Conv. Internaz. di Teoria dei Gruppi Finiti, Firenze, 1960, Edizioni Cremonese, Rome, 1960.

5. L. G. Kovács and M. F. Newman, Cross varieties of groups, Proc. Roy. Soc. Ser. A 292 (1966), 530-536.

6. R. C. Lyndon, Two notes on nilpotent groups, Proc. Amer. Math. Soc. 3 (1952), 579-583.

7. B. H. Neumann, Identical relations in groups. I, Math. Ann. 114 (1937), 505525.

8. Sheila Oates and M. B. Powell, Identical relations in finite groups, J. Algebra 1 (1964), 11-39.

Graduate Center, The City University of New York 